\title{
Apatinib mesylate tablet in the treatment of advanced malignant melanoma
}

This article was published in the following Dove Press journal:

OncoTargets and Therapy

\author{
Lingge Yang ${ }^{1,2, *}$ \\ Huiyan Zhu',2,* \\ Peng Luo ${ }^{1,2}$ \\ Shiqi Chen ${ }^{1,2}$ \\ Yu Xu ${ }^{1,2}$ \\ Chunmeng Wang ${ }^{1,2}$ \\ 'Department of Bone and Soft Tissue \\ Sarcomas, Fudan University Shanghai \\ Cancer Center, Shanghai, People's \\ Republic of China; ${ }^{2}$ Department of \\ Oncology, Shanghai Medical College, \\ Fudan University, Shanghai, People's \\ Republic of China \\ *These authors contributed equally \\ to this work
}

Background: Observing and studying clinical efficacy and safety of apatinib mesylate tablet in the treatment of advanced malignant melanoma (MM).

Methods: Retrospectively analyzing the clinical data of 22 patients with metastatic MM who had failed conventional chemotherapy from June 2016 to January 2018. All patients took $500 \mathrm{mg}$ of apatinib mesylate tablets per day. The efficacy should be evaluated according to RECIST 1.1 criteria. Adverse events (AEs) should be graded according to NCI-CTCAE 4.0.

Results: There were two cases of partial remission (PR), 11 of stable disease (SD) and nine of progressive disease (PD) in the 22 patients with advanced MM, where the objective remission rate (ORR) was $9.1 \%$ and the disease control rate (DCR) was $59.1 \%$. The median progressionfree survival (PFS) was 7.5 months, and the 6-month progression-free survival rate (PFR) was $54.7 \%$. Six patients died and the overall survival (OS) was not reached. AEs were controllable and all were in Grade 1-3.

Conclusion: Apatinib mesylate tablets have a certain curative effect on patients with malignant melanomas of Stage IV who failed conventional chemotherapy. Apatinib mesylate tablets at a daily dose of $500 \mathrm{mg}$ are well tolerated by most patients.

Keywords: apatinib, malignant melanoma, targeted therapy, adverse reaction, efficacy

\section{Plain language summary}

In this work, we assessed retrospectively clinical efficacy and safety of apatinib mesylate tablet in the treatment of 22 patients with advanced malignant melanoma of Stage IV at a single institution. Meanwhile, we found that apatinib had a certain curative effect on those patients and it was well tolerated by most patients. Moreover, we believe that in the near future, apatinib is expected to become a new option for treating patients with advanced malignant melanoma.

\section{Background}

Malignant melanoma (MM) is a highly malignant tumor that originates in the neural crest melanocyte and is caused by hyperproliferation of abnormal melanocytes. Clinically, MM mostly originates in skin, mucous membrane and vascular coat of eyeball uvea. As a highly invasive type of tumor, lymph and hematogenous metastasis may occur early in tumor formation with a poor prognosis and the 5-year survival rate is $10 \% .{ }^{1}$ It is one of the malignant tumors that seriously threaten human health. For patients with Stage IV MM, systemic treatment is mainly medical treatment in general, including chemotherapy, targeted therapy, and immunotherapy, etc. Among them, chemotherapy drugs are mainly based on a combination of DTIC ${ }^{\circledR}$ (dacarbazine), Temodal ${ }^{\circledR}$ (temozolomide, TMZ), platinum salts and taxanes. Targeted therapy is represented by Glivec ${ }^{\circledR}$ (imatinib), Zelboraf ${ }^{\circledR}$ (vemurafenib), and Avastin ${ }^{\circledR}$ (bevacizumab). Traditional immunotherapy is represented by high-dose Interleukin-2
Correspondence: Chunmeng Wang Department of Bone and Soft Tissue Sarcomas, Fudan University Shanghai Cancer Center; Department of Oncology, Shanghai Medical College, Fudan University, No. 270, Dong'an Road, Xuhui District, Shanghai 200032, People's Republic of China Fax +86 2164430130 Email cmwang1975@163.com

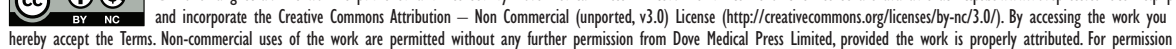
hereby accept the Terms. Non-commercial uses of the work are permitted without any further permission from Dove Medicat 
(IL-2). In recent years, targeted immune therapy drugs, such as Yervoy $^{\circledR}$ (ipilimuab), Keytruda ${ }^{\circledR}$ (pembrolizumb), Opdivo $^{\circledR}$ (nivolumab) and so on, have yielded unusually brilliant results, providing a new way for MM treatment. In addition, in order to search more safe and effective systemic treatment approaches, multiple treatments are being explored.

Apatinib mesylate tablet (Apatinib) is a new generation of oral small molecule anti-angiogenesis inhibitor, the main mechanism of action of which is a competitive system of binding where vascular endothelial growth factor receptor-2 (VEGFR-2) and receptor-intracellular tyrosine ATP binding site highly selectively inhibit the activity of VEGFR-2 tyrosine kinase and block the signal transduction after the combination of vascular endothelial growth factors (VEGF), thereby strongly inhibiting tumor angiogenesis. It has been proved to have a good curative effect on gastric cancer. ${ }^{2}$ In the advanced MM, antiangiogenic therapy is still an important therapeutic method. Related studies using chemotherapy combined with recombinant human Endostar ${ }^{\mathrm{TM}}$ (endostatin), ${ }^{3}$ or monoclonal antibody drugs (McAb) of VEGFR, ${ }^{4-6}$ have confirmed that chemotherapy combined with antiangiogenic therapy can improve the survival of patients with advanced MM. Therefore, in our study, apatinib mesylate tablet was used as the main drug to treat patients with Stage IV MM who failed conventional chemotherapy, in order to evaluate the clinical efficacy and safety of the drug.

\section{Methods}

\section{Patients}

From June 2016 to January 2018, we collected 22 cases of Stage IV MM patients who underwent at least one conventional chemotherapy treatment which failed in the Department of Bone and Soft Tissue Sarcomas at Fudan University Shanghai Cancer Center, including 15 cases of males and seven females, aged from 28 to 81 , with a median age of 51. These MM patients were examined and confirmed through histopathology that they were not suitable for surgical treatment and their clinical stage was IV. In addition, the curative effect of their lesions could be evaluated by imaging studies. The study was approved by the Ethics Committee of Fudan University Shanghai Cancer Center and all aspects of the study comply with the Declaration of Helsinki. The Ethics Committee of Fudan University Shanghai Cancer Center specifically approved that no informed consent was required because of the retrospective nature of the study. Data of all patients participating in this study were confidential and the patients participating in the study did so completely voluntarily and were not forced to participate.

\section{Treatment}

All patients took $500 \mathrm{mg}$ (two tablets) orally of apatinib mesylate tablets once a day, half an hour after meals for treatment and the treatment cycle was 4 weeks. Adverse events (AEs) were evaluated, graded and recorded according to the National Cancer Institute-Common Terminology Criteria for Adverse Events (NCI-CTCAE) Version 4.0. Patients with AEs Grade 1-2 were recommended to continue medication with clinical follow-up observations. For patients with AEs Grade 3 and above, they should suspend medication and should be hospitalized for symptomatic treatment. When the AEs restore to $\leq$ Grade 2, these patients could continue receiving treatment if the medicine was well tolerated. If it was estimated that the patients were to be severely harmed or might have severe sequelae, the medicine should be stopped.

\section{Assessment}

Baseline assessment included detailed medical history, physical examination, routine laboratory examination, and computed tomography (CT) or magnetic resonance imaging (MRI) scans of the primary or metastatic sites. The efficacy was to be evaluated after each cycle of treatment according to Response Evaluation Criteria in Solid Tumors (RECIST) Version 1.1, and it was categorized into complete remission (CR), partial remission (PR), stable disease (SD), and progressive disease (PD). Objective remission rate $(\mathrm{ORR})$ was based on $\mathrm{CR}+\mathrm{PR}$ calculation and disease control rate (DCR) was based on $\mathrm{CR}+\mathrm{PR}+\mathrm{SD}$ calculation. Progression-free survival (PFS) was defined as the time from the start of treatment with apatinib to PD or death, and overall survival (OS) as the time between the start of treatment and the death of the patients. Follow-ups were conducted up to January 31, 2018.

\section{Statistical methods}

For analyzing the efficacy and safety of apatinib, all patients were treated with it for more than 1 cycle in this study. All data were analyzed statistically using SPSS Version 23.0 software. Survival analysis adopted Kaplan-Meier method.

\section{Results}

\section{Characteristics of patients at baseline}

Among the 22 patients, there were 12 cases of MM derived from limbs, four from anal canal, three from vulva, one from mammary gland and two of unknown origin. All 
were clinically in Stage IV, based on the staging system of American Joint Committee on Cancer (AJCC) eighth edition, including seven cases in Stage M1a, five in M1b and 10 in M1c. Common metastatic sites included nine cases of distant lymph node, nine of the lung, six of the liver, three of the abdominal cavity, one of spleen and one of kidney. The patients had failed all the previous chemotherapy. The chemotherapy was a conventional one with DTIC or TMZbased single drug, or combined cisplatin and endostatin. The course of treatment was 1-10 cycles and the median was four cycles. In addition, all the patients had good main organ function, normal blood, urine and stool routine, with no basic diseases like previous cardiovascular diseases such as hypertension, gastrointestinal diseases, and hand-foot skin reaction, etc, which were difficult to distinguish from the medicine AEs. See Table 1 for details.

Table I Patients' characteristics ( $\mathrm{N}=22)$

\begin{tabular}{|c|c|c|}
\hline Characteristics & $\begin{array}{l}\text { No of } \\
\text { patients }\end{array}$ & $\%$ of total \\
\hline \multicolumn{3}{|l|}{ Gender } \\
\hline Male & 15 & 68.2 \\
\hline Female & 7 & 31.8 \\
\hline Median age (years, range) & 51 & $28-81$ \\
\hline \multicolumn{3}{|l|}{ ECOG performance status } \\
\hline 0 & 5 & 22.7 \\
\hline I & 17 & 77.3 \\
\hline \multicolumn{3}{|l|}{ Primary tumor site } \\
\hline Extremity & 12 & 54.6 \\
\hline Anal canal & 4 & 18.2 \\
\hline Vulva & 3 & 13.6 \\
\hline Breast & 1 & 4.5 \\
\hline Unknown & 2 & 9.1 \\
\hline \multicolumn{3}{|l|}{ Metastatic sites } \\
\hline Lymph node & 9 & 40.9 \\
\hline Lung & 9 & 40.9 \\
\hline Liver & 6 & 27.3 \\
\hline Intraperitoneal & 3 & 13.6 \\
\hline Spleen & 1 & 4.5 \\
\hline Kidney & 1 & 4.5 \\
\hline \multicolumn{3}{|l|}{ Type of metastatic site } \\
\hline Nonvisceral & 7 & 31.8 \\
\hline Visceral & 15 & 68.2 \\
\hline \multicolumn{3}{|l|}{ No of metastatic sites } \\
\hline I & 9 & 40.9 \\
\hline 2 & 5 & 22.7 \\
\hline$\geq 3$ & 8 & 36.4 \\
\hline \multicolumn{3}{|l|}{ Prior chemotherapy } \\
\hline $\mathrm{TMZ}$ & 3 & 13.6 \\
\hline DTIC + DDP & 3 & 13.6 \\
\hline DTIC + DDP + Endostar & 16 & 72.8 \\
\hline \multicolumn{3}{|l|}{ Chemotherapy period } \\
\hline$<4$ cycles & 5 & 22.7 \\
\hline 4-6 cycles & 12 & 54.6 \\
\hline$>6$ cycles & 5 & 22.7 \\
\hline
\end{tabular}

Abbreviations: No, number; ECOG, Eastern Cooperative Oncology Group; TMZ, temozolomide; DTIC, dacarbazine; DDP, cisplatin.
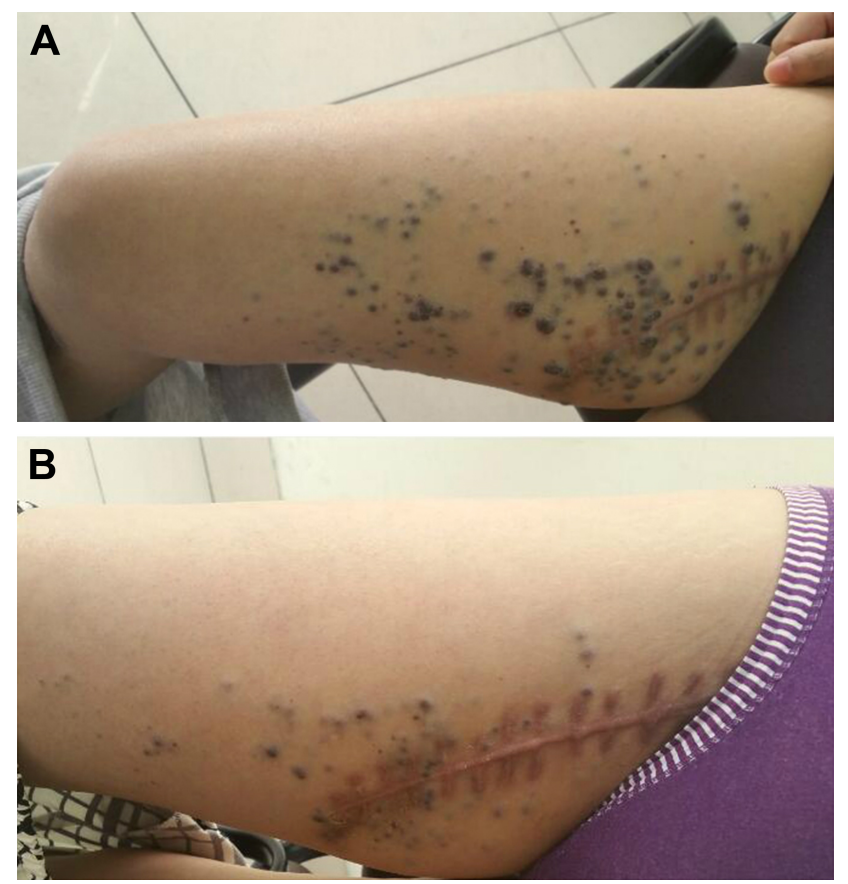

Figure I (A) The primary lesions before (B) and after the four courses of medication.

\section{Efficacy}

As of January 2018, 22 patients had received follow-up visits in the efficacy evaluation phase (medicine taken for more than one cycle). The follow-up time ranged from 2.2 to 15.8 months, and the median was 9.2 months. Among them, two patients got PR (as show in Figure 1), 11 got SD (as shown in Figure 2), and nine got PD. The ORR was 9.1\% and the DCR was $59.1 \%$. The median PFS was 7.5 months (95\% of CI was 4.4-10.6 months, as shown in Figure 3), and the 6-month PFR was $54.7 \%$. Six patients died and the median OS was not reached.

\section{Toxicity}

All the patients in the group tolerated apatinib well at a dose of $500 \mathrm{mg}$ per day, and AEs, all in Grade 1-3, were controllable, with no drug-related AEs of Grade 4 and above. The most common AEs were gastrointestinal reactions (mainly abdominal distension, anorexia, nausea, vomiting) and fatigue, and the incidence was 50\%. Other AEs were high blood pressure, palpitation and other cardiovascular symptoms, with an incidence of $40.9 \%$. Grade 3 AEs were high blood pressure, a total of three cases, and the incidence was 13.6\%. See Table 2 for details.

\section{Discussion}

According to the data released by WHO in 2013, there are about 200,000 new cases of MM in the world each year, 


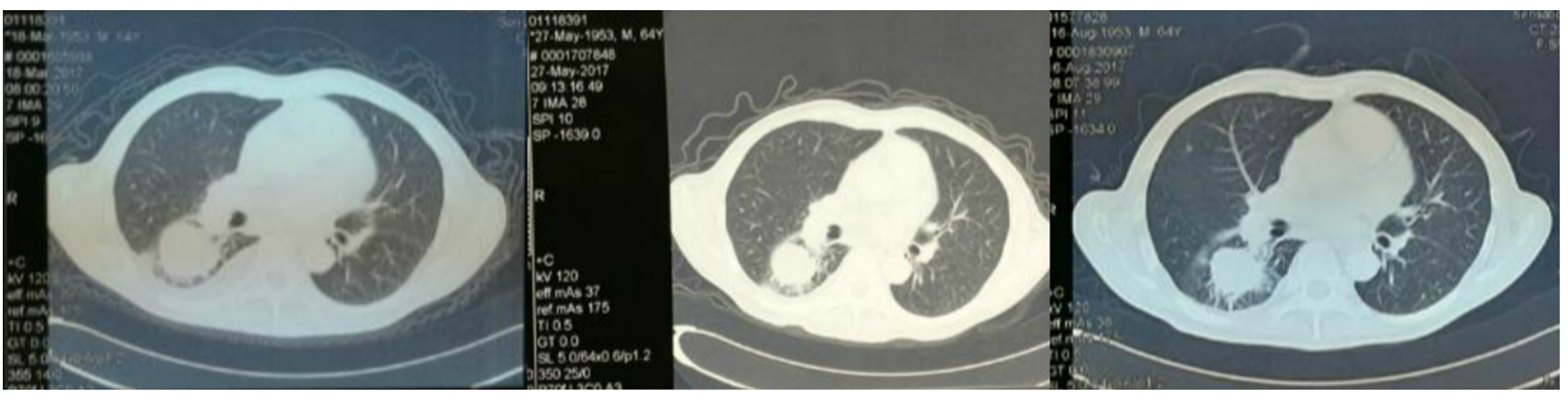

Figure 2 The computed tomography images of metastatic sites at the start of treatment (Left), after two courses of medication (Center) and four courses of medication (Right).

causing about 65,000 deaths, ${ }^{7}$ with the features of high metastasis, high mortality, and strong resistance. The main cause of death of MM is distant metastasis, including lung, liver, bone, and brain metastasis. ${ }^{8}$ Metastasis occurs in about $20 \%{ }^{9}$ of the patients at the first diagnosis of MM and those patients have been in the advanced stage when a definite diagnosis is made. Those with metastasis to skin, subcutaneous tissue or lymphoid tissue have relatively better prognosis. The prognosis of metastasis to the lung is medium, and if metastasis is transferred to liver, bone, brain, or sites with higher lactate dehydrogenase (LDH), the prognosis is relatively poor. Advanced MM progresses rapidly and has a poor prognosis.

Previous treatments have been based on DTIC, TMZ, platinum salt and taxanes for chemotherapy for a long time. However, in a randomized controlled trial of Phase III, the ORR of the DTIC + placebo group was only $10.3 \%$ (26/252), DCR was $30.2 \%$, and the median OS was only 9.1 months. ${ }^{10}$ TMZ is a small molecule oral agent of DTIC analogs. It is a new type of the second-generation alkylating agent the efficacy

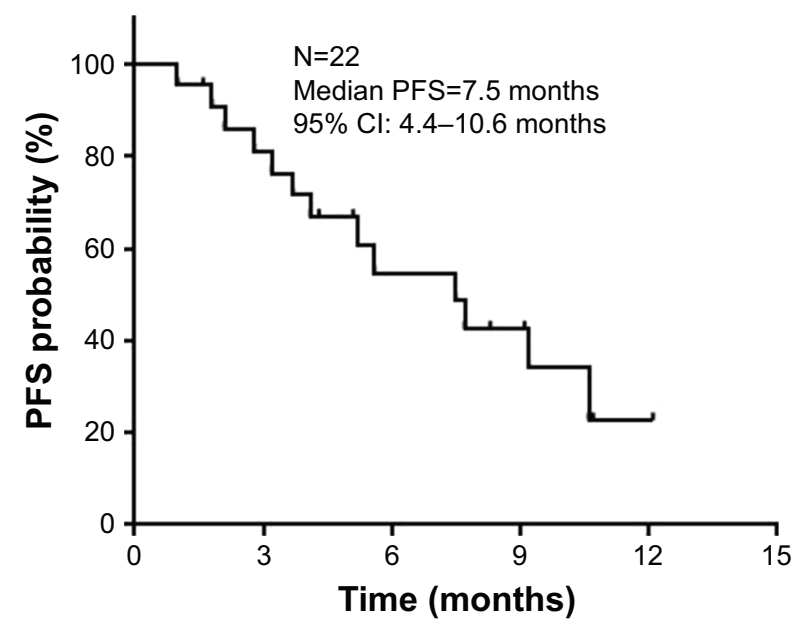

Figure 3 Kaplan-Meier plot of PFS for all patients. Abbreviation: PFS, progression-free survival. of which is similar to that of DTIC. Since TMZ does not need to be metabolized through liver and can pass through the bloodbrain barrier, reducing the physiological damage of patients and improving their living quality, it is often used as a first-line treatment for patients with advanced MM. ${ }^{11}$ In this research, the patients all failed previous chemotherapy regimens of DTIC or TMZ-based single agent or conventional chemotherapy combined with cisplatin and endostatin, with a treatment course of 1-10 cycles and a median duration of 4 cycles.

Due to the presence of genetic mutations in many MM patients, including BRAF, NRAS, KIT, PTEN, GNAQ, GNA11, and CDKN2A, ${ }^{12,13}$ immunotherapy and targeted therapy progress rapidly in the advanced MM field. Multiple treatment methods are being explored in order to search safer and more effective systemic treatment approaches. For MM patients with KIT mutation, imatinib is a good treatment option. In a Phase II clinical trial study, 24 patients with metastatic MM were treated with imatinib, with an ORR of $29 \%$, a DCR of $50 \%$, a median PFS of 3.7 months and a median

Table 2 AEs experienced by the patients $(\mathrm{N}=22)$

\begin{tabular}{|c|c|c|c|c|c|c|}
\hline \multirow[t]{3}{*}{ AEs } & \multicolumn{4}{|c|}{ Grade } & \multicolumn{2}{|c|}{ Total } \\
\hline & \multicolumn{2}{|c|}{ I or 2} & \multicolumn{2}{|c|}{3 or 4} & \multirow[b]{2}{*}{ No } & \multirow[b]{2}{*}{$\%$} \\
\hline & No & $\%$ & No & $\%$ & & \\
\hline \multicolumn{7}{|l|}{ Non-hematologic } \\
\hline Gastrointestinal symptoms ${ }^{a}$ & II & 50 & 0 & 0 & II & 50 \\
\hline Fatigue & 11 & 50 & 0 & 0 & 11 & 50 \\
\hline Hypertension & 6 & 27.3 & 3 & 13.6 & 9 & 40.9 \\
\hline Palpitation & 4 & 18.2 & 0 & 0 & 4 & 18.2 \\
\hline Rash & 3 & 13.6 & 0 & 0 & 3 & 13.6 \\
\hline Hand-foot syndrome & 3 & 13.6 & 0 & 0 & 3 & 13.6 \\
\hline Proteinuria & 3 & 13.6 & 0 & 0 & 3 & 13.6 \\
\hline Liver dysfunction & 1 & 4.5 & 0 & 0 & I & 4.5 \\
\hline \multicolumn{7}{|l|}{ Hematologic } \\
\hline Leukopenia & 3 & 9.1 & 0 & 0 & 3 & 13.6 \\
\hline Thrombocytopenia & 2 & 9.1 & 0 & 0 & 2 & 9.1 \\
\hline
\end{tabular}

Note: a Gastrointestinal symptoms include abdominal distention, anorexia, nausea, vomiting, etc.

Abbreviation: AEs, adverse events. 
OS of 12.5 months. ${ }^{14}$ When nilotinib, also as KIT inhibitor, combines with DTIC, it will have similar efficacy to that of imatinib in advanced MM. ${ }^{15}$ A large-scale analysis showed that the gene mutation rate of BRAF in Chinese MM patients was about $25.5 \% .{ }^{16}$ This proportion was significantly lower than that of Caucasians. Even so, BRAF inhibitor is of very important significance to the MM treatment in China. Vemurafenib is a member of the BRAF inhibitors. In a multicenter Phase III randomized controlled trial study, the ORR of the vemurafenib group was as high as $48.4 \%$, while that of the control group was only $5.5 \%$ and all subgroup analyses demonstrated that the PFS and OS of the vemurafenib group were significantly improved compared with the DTIC group, and the hazard ratios were 0.26 and 0.37 respectively. ${ }^{17}$ In addition, immunosuppression inhibitors have yielded unusually brilliant results in recent years, significantly prolonging patients' OS. Among them, ipilimuab (anti-CTLA-4 McAb), ${ }^{10}$ pembrolizumb (anti-PD-1 McAb), ${ }^{18}$ and nivolumab (antiPD-1 McAb), ${ }^{19}$ have been approved by the Food and Drug Administration in the United States (US-FDA) for treating advanced unresectable or metastatic MM.

In MM, anti-angiogenic therapy remains an important therapeutic target for advanced MM. A Phase II clinical trial shows that combining endostatin with DTIC first-line treatment of advanced MM, compared with DTIC monotherapy, can significantly prolong PFS (4.5 vs 1.5 months, $P=0.013$ ) and $\mathrm{OS}$ (12.0 vs 8.0 months, $P=0.005)$, with good tolerance. ${ }^{3}$ Another type of monoclonal antibody, bevacizumab, selectively combines human vascular endothelial growth factor (VEGF). The efficacy of bevacizumab uniting chemotherapy has also been demonstrated in advanced MM. ${ }^{46}$ For example, in a clinical Phase II randomized controlled trial, combining bevacizumab with carboplatin and paclitaxel chemotherapy medicine in the control group for treatment, the ORR was 13\%, the median PFS was 5.6 months, and the median OS was 14.5 months, and when erolimus was added to the experimental group, the ORR could be increased to $23 \%$, while the median PFS and the median OS were not as good as those in the control group. ${ }^{6}$ The above research indicated that antiangiogenic inhibitors may be effective in MM treatment. Therefore, we used Apatinib Mesylate Tablet, a novel small-molecule inhibitor of VEGFR-2 tyrosine kinase, for treating the metastatic MM. Apatinib has potent antiangiogenic effect. Compared with similar small molecule tyrosine kinase inhibitors, it has higher selectivity and stronger inhibitory effect on VEGFR-2. As confirmed in Phase III controlled study, it has been approved for being used in advanced gastric carcinoma. ${ }^{20}$
Through retrospective analysis, among the 22 patients in the group, two got PR, 11 got SD and nine got PD. With the data analysis, the ORR in our series was 9.1\%, and the DCR was $59.1 \%$. The time of follow-up visits ranged from 2.2 to 15.8 months, the median was 9.2 months, the median PFS was 7.5 months (95\% of CI was 4.4-10.6 months), the 6-month PFR was 54.7\%. However, the median OS was not reached. Compared to all the previous studies mentioned above, ORR is comparable to conventional chemotherapeutic drugs and other anti-angiogenic drugs, but is not as good as the medicine of targeted therapy and immunotherapy, while DCR is higher than conventional chemotherapeutic drugs, slightly higher than that of targeted therapy and immunotherapy. Compared with all the abovementioned drugs, apatinib can prolong the patient's PFS for at least 1 month, which has good clinical application value.

In terms of safety, the entire group of patients tolerated the daily dose of $500 \mathrm{mg}$ of apatinib well, and AEs were controllable, all of which were only in Grade 1-3. No drug-related AEs of Grade 4 and above appeared. The main AEs were gastrointestinal reactions (abdominal distension, anorexia, nausea, vomiting, etc.), fatigue, hypertension, palpitation, hand-foot skin reaction (hand-foot syndrome, rash, etc.), proteinuria, impaired liver function and bone marrow suppression (mainly leukocytopenia and thrombocytopenia), etc., and this is similar to the related reports. ${ }^{20,21}$ Among them, AEs of Grade 3 were hypertension, and there were three cases in total. The incidence rate was $13.6 \%$. Patients who suspended medication or were hospitalized for symptomatic treatment for 2-3 days could all recover to Grade 2 or below and continued medication. All patients continued medication until PD or death. Overall, the daily dose of $500 \mathrm{mg}$ of apatinib treating patients with advanced MM in our group was well tolerated.

This study is a retrospective analysis and has certain limitations. First, only 22 cases were analyzed, and there was a certain treatment selection bias. Second, there was no control group so that no relevant hypothesis test could be performed. Furthermore, the follow-up time was insufficient and the median OS was not reached. But estimated with a median PFS of 7.5 months and a median follow-up time of 9.2 months, apatinib is a good prospect for prolonging patients' OS. Finally, due to the insufficient sample size, the safety of the medicine was not well reflected. Roviello et $\mathrm{al}^{2}$ reviewed the efficacy and safety of apatinib in gastric cancer, and found that the incidence of AEs in Grade 3-4 was just about $2 \%$. 


\section{Conclusion}

In conclusion, when apatinib mesylate tablet is used to treat advanced MM at a dose of $500 \mathrm{mg}$ per day, the overall tolerability of the patients is good. From the patients who failed conventional chemotherapy, a relatively ideal therapeutic effect has been observed. The efficacy of further clinical trials is worth considering, and apatinib's AEs need to be further observed. It is believed that in the near future, apatinib is expected to become a new option for treating patients with advanced MM.

\section{Data sharing statement}

The datasets used and/or analyzed during the current study are available from the corresponding author on reasonable request. The data of patients participating in this study is confidential and patients participating in the study were completely voluntary, and were not forced to participate.

\section{Acknowledgments}

We thank the patients who shared their experiences with our oncologists. No funding was received.

\section{Disclosure}

The authors report no conflicts of interest in this work.

\section{References}

1. Redman JM, Gibney GT, Atkins MB. Advances in immunotherapy for melanoma. BMC Med. 2016;14(1):20.

2. Roviello G, Ravelli A, Polom K, et al. Apatinib: a novel receptor tyrosine kinase inhibitor for the treatment of gastric cancer. Cancer Lett. 2016;372(2):187-191.

3. Cui C, Mao L, Chi Z, et al. A phase II, randomized, double-blind, placebo-controlled multicenter trial of Endostar in patients with metastatic melanoma. Mol Ther. 2013;21(7):1456-1463.

4. Kottschade LA, Suman VJ, Perez DG. A randomized phase 2 study of temozolomide and bevacizumab or nab-paclitaxel, carboplatin, and bevacizumab in patients with unresectable stage IV melanoma. Cancer. 2013;119(3):586-592.

5. Spitler LE, Boasberg P, O’Day S, et al. Phase II study of nab-paclitaxel and bevacizumab as first-line therapy for patients with unresectable stage III and IV melanoma. Am J Clin Oncol. 2015;38(1):61-67.
6. McWilliams RR, Allred JB, Slostad JA, et al. NCCTG N0879 (Alliance): a randomized phase 2 cooperative group trial of carboplatin, paclitaxel, and bevacizumab \pm everolimus for metastatic melanoma. Cancer. 2018;124(3):537-545.

7. Freeman-Keller M, Weber JS. Anti-programmed death receptor 1 immunotherapy in melanoma: rationale, evidence and clinical potential. Ther Adv Med Oncol. 2015;7(1):12-21.

8. Zhu Z, Liu W, Gotlieb V. The rapidly evolving therapies for advanced melanoma - Towards immunotherapy, molecular targeted therapy, and beyond. Crit Rev Oncol Hematol. 2016;99:91-99.

9. Lindsay CR, Spiliopoulou P, Waterston A. Blinded by the light: why the treatment of metastatic melanoma has created a new paradigm for the management of cancer. Ther Adv Med Oncol. 2015;7(2):107-121.

10. Robert C, Thomas L, Bondarenko I, et al. Ipilimumab plus dacarbazine for previously untreated metastatic melanoma. $N$ Engl J Med. 2011; 364(26):2517-2526.

11. Middleton MR, Grob JJ, Aaronson N, et al. Randomized phase III study of temozolomide versus dacarbazine in the treatment of patients with advanced metastatic malignant melanoma. J Clin Oncol. 2000; 18(1):158-166.

12. Zebary A, Omholt K, Vassilaki I, et al. KIT, NRAS, BRAF and PTEN mutations in a sample of Swedish patients with acral lentiginous melanoma. J Dermatol Sci. 2013;72(3):284-289.

13. van Raamsdonk CD, Griewank KG, Crosby MB, et al. Mutations in GNA11 in uveal melanoma. N Engl J Med. 2010;363(23):2191-2199.

14. Hodi FS, Corless CL, Giobbie-Hurder A, et al. Imatinib for melanomas harboring mutationally activated or amplified KIT arising on mucosal, acral, and chronically sun-damaged skin. J Clin Oncol. 2013; 31(26):3182-3190.

15. Guo J, Carvajal RD, Dummer R, et al. Efficacy and safety of nilotinib in patients with KIT-mutated metastatic or inoperable melanoma: final results from the global, single-arm, phase II TEAM trial. Ann Oncol. 2017;28(6):1380-1387.

16. Si L, Kong Y, Xu X, et al. Prevalence of BRAF V600E mutation in Chinese melanoma patients: large scale analysis of BRAF and NRAS mutations in a 432-case cohort. Eur J Cancer. 2012;48(1):94-100.

17. Chapman PB, Hauschild $A$, Robert $C$, et al. Improved survival with vemurafenib in melanoma with BRAF V600E mutation. $N$ Engl J Med. 2011;364(26):2507-2516.

18. Ribas A, Hamid O, Daud A, et al. Association of pembrolizumab with tumor response and survival among patients with advanced melanoma. JAMA. 2016;315(15):1600-1609.

19. Robert C, Long GV, Brady B, et al. Nivolumab in previously untreated melanoma without BRAF mutation. $N$ Engl J Med. 2015;372(4): 320-330.

20. Li J, Qin S, Xu J, et al. Randomized, double-blind, placebo-controlled phase III trial of apatinib in patients with chemotherapy-refractory advanced or metastatic adenocarcinoma of the stomach or gastroesophageal junction. J Clin Oncol. 2016;34(13):1448-1454.

21. Hu X, Zhang J, Xu B, et al. Multicenter phase II study of apatinib, a novel VEGFR inhibitor in heavily pretreated patients with metastatic triple-negative breast cancer. Int J Cancer. 2014;135(8):1961-1969.
OncoTargets and Therapy

\section{Publish your work in this journal}

OncoTargets and Therapy is an international, peer-reviewed, open access journal focusing on the pathological basis of all cancers, potential targets for therapy and treatment protocols employed to improve the management of cancer patients. The journal also focuses on the impact of management programs and new therapeutic agents and protocols on

\section{Dovepress}

patient perspectives such as quality of life, adherence and satisfaction The manuscript management system is completely online and includes a very quick and fair peer-review system, which is all easy to use. Visit http://www.dovepress.com/testimonials.php to read real quotes from published authors. 